\title{
Erratum to: 6-week radiographs unsuitable for diagnosis of suspected scaphoid fractures
}

\author{
Wouter H. Mallee ${ }^{1}$ (i) $\cdot$ Jos J. Mellema ${ }^{2} \cdot$ Thierry G. Guitton $^{3} \cdot$ J. Carel Goslings ${ }^{4}$. \\ David Ring ${ }^{5}$ Job N. Doornberg ${ }^{1} \cdot$ Science of Variation Group
}

Published online: 10 March 2017

(C) Springer-Verlag Berlin Heidelberg 2017

\section{Erratum to: Arch Orthop Trauma Surg (2016) 136:771-778 DOI 10.1007/s00402-016-2438-4}

Due to an error during the production process, the HTML version of this article does not contain all information of the members of the Science of Variation Group. The members of the Science of Variation Group are:

Paul A. Martineau, Asif Ilyas, Brian P. D. Wills, C. Taleb, Camilo Jose Romero Barreto, Cesar dario Oliveira Miranda, C. H. Fernandes, Chad Manke, Charles A. Goldfarb, Christopher J. Walsh, Christopher M. Jones, Constanza L. Moreno-Serrano, Daniel A. Osei, Daniel Polatsch, David M. Kalainov, Eric Hofmeister, E. T. Walbeehm, Evan D. Schumer, F. Thomas D. Kaplan, Fabio Suarez, Frank L. Walter, G. A. Kraan, Gary M. Pess, George W. Balfour, Hervey L. Kimball, H. W. Grunwald, Jack Choueka, J. E. Foriguq, Jeffrey A. Greenberg, Jeffrey

The online version of the original article can be found under doi:10.1007/s00402-016-2438-4.

Wouter H. Mallee

w.h.mallee@amc.uva.nl

1 Department of Orthopedic Surgery, Academic Medical Center Amsterdam, AMC, Meibergdreef 9, 1105 AZ Amsterdam, The Netherlands

2 Hand and Upper Extremity Service, Massachusetts General Hospital, Boston, MA, USA

3 Department of Plastic Surgery, University Medical Center Groningen, Groningen, The Netherlands

4 Department of Trauma Surgery, Academic Medical Center Amsterdam, Amsterdam, The Netherlands

5 Department of Surgery and Perioperative Care, Dell Medical School, Austin, USA
Wint, John M. Erickson, John McAuliffe, John T. Capo, John Taras, Jose A. Ortiz, Julie Adams, Karl-Josef Prommersberger, Kevin M. Rumball, Kyle D. Bickel, Lior Paz, Lisa Lattanza, Louis Catalano III, M. Jason Palmer, Marc J. Richard, Marco Rizzo, Maurizio Calcagni, Maximillian Soong, Megan M. Wood, Michael Baskies, Michael Behrman, Michael Darowish, Michael Nancollas, Michael W. Grafe, Michael W. Kessler, Miguel A. Pirela-Cruz, M. P. Bekerom, Naquira Escobar Luis Felipe, Nathan Hoekzema, Oleg M. Semenkin, Patrick W. Owens, Philip Blazar, Ralph M Costanzo, Ramon de Bedout, Renato M. Fricker, Richard L. Hutchison, Richard S. Gilbert, Rick Papandrea, Robert R. Slater, Robert R. L. Gray, Ryan Klinefelter, Ryan P. Calfee, Sander Spruijt, Sanjeev Kakar, Saul Kaplan, Seth Dodds, Stephen A. Kennedy, Steven Beldner, T. Apard, Taizoon Baxamusa, Thomas G. Stackhouse, Todd Siff, W. Arnnold Batson, Warren C. Hammert. 